\title{
Análisis de Costos en la Producción de Quesos Artesanales. Estudio de Caso: Quesería ell Bejucal
}

\author{
Cost Analysis in the Production of Artisan Cheeses. Case \\ Study: Quesería el Bejucal
}
Paredes-Maas, Ezequiel; Monroy-Hernández, Rubén; Chávez-Dehesa, Juan Francisco; Guevara-Hernández, Francisco; Castro-Ehuan, Emilio; Zaldívar-Cruz, Juan Manuel; Editor Academico Prof. M.Sc Adelfa Patricia Colón-García

\author{
Ezequiel Paredes-Maas \\ ezeparedes@hotmail.com \\ Universidad Tecnológica del Usumacinta, México \\ Rubén Monroy-Hernández \\ ruben.monroy@unach.mx \\ Facultad Maya de Estudios Agropecuarios de la \\ UNACH, México \\ Juan Francisco Chávez-Dehesa \\ jfcdehesa@hotmail.com \\ Universidad Tecnológica del Usumacinta, México \\ Francisco Guevara-Hernández \\ francisco.guevaramxnl@gmail.com \\ Universidad Autónoma de Chiapas (UNACH)., \\ México \\ Emilio Castro-Ehuan \\ ecastroehuan@hotmail.com \\ Profesionista egresado de la M.T. en Productos lácteos \\ del COLPOS. Adscrito a la "Quesería el Bejucal", \\ México \\ Juan Manuel Zaldívar-Cruz \\ Colegio de Posgraduados, Campus, Cárdenas, Tab, \\ México
}

Editor Academico Prof. M.Sc Adelfa Patricia Colón-

García

Universidad Nacional Autonoma de Honduras

CURLA, Honduras

Revista Iberoamericana de Bioeconomía y Cambio Climático

Universidad Nacional Autónoma de Nicaragua, León, Nicaragua ISSN-e: 2410-7980

Periodicidad: Semestral

vol. 5, núm. 10, 2019

czuniga@ev.unanleon.edu.ni

Recepción: 15 Julio 2019

Aprobación: 04 Diciembre 2019

URL: http://portal.amelica.org/ameli,

jatsRepo/394/3941757002/index.html
Resumen: La actividad económica resultado de la elaboración de quesos artesanales, es de gran importancia para la economía local, no existe un estudio de este tipo que permita a los productores conocer a detalle los costos de manufactura del queso artesanal, actualmente los productores sólo tienen aproximaciones y sus precios de venta los determinan de acuerdo al comportamiento del mercado, sin tener certeza acerca del margen de utilidad. Por ello, se realizó este trabajo que consistió en determinar la estructura de costos en cada tipo de queso artesanal, que se elabora en la Quesería “El Bejucal”. Esta empresa es una de las más emblemáticas debido a su tradición y prestigio, ubicada en el municipio de Balancán, Tabasco, y cuya marca está protegida ante el Instituto Mexicano de la Propiedad Industrial (IMPI). Se realizaron cálculos basados en la clasificación de costos directos, indirectos y gastos de administración y ventas. Se encontró que el queso de poro es el producto que más alto costo de producción tiene, respecto a los demas productos, debido principalmente al elevado costo del material de empaque. Sin embargo, el ingreso por la comercialización del queso de poro corresponde al 70\% y el restante a los otros quesos; el queso de poro es de pasta friable,blanda y prensada; ligeramente madurado, elaborado a partir de leche bronca, se comercializa en piezas de aproximadamente $300 \mathrm{~g}$.

Palabras clave: Estructura de costos, Quesos artesanales, Margen de utilidad.

Abstract: The economic activity resulting from the production of artisan cheeses, is of great importance for the local economy, there is no such study that allows producers to know in detail the manufacturing costs of artisan cheese, currently producers only have approximations and their sales prices determine them according to the behavior of the market, without being certain about the profit margin. Therefore, this work was carried out that consisted in determining the cost structure in each type of artisanal cheese, which is elaborated in the "El Bejucal" cheese factory. This company is one of the most emblematic due to its tradition and prestige, located in the municipality of Balancán, Tabasco, and whose brand is protected by the Mexican Institute of Industrial Property (IMPI). Calculations were made based on the classification of direct and indirect costs and administration 
DOI: https://doi.org/10.5377/ribcc.v5i10.8969

Autor de correspondencia: ruben.monroy@unach.mx and sales expenses. It was found that pore cheese is the product with the highest production cost, compared to other products, mainly due to the high cost of packaging material. However, the income from the commercialization of the pore cheese corresponds to $70 \%$ and the rest to the other cheeses; the pore cheese is friable, soft and pressed pasta; slightly ripened, made from raw milk, is sold in pieces of approximately $300 \mathrm{~g}$.

Keywords: Cost structure, Artisan cheeses, Profit margin.

\section{INTRODUCCIÓN}

El queso de poro se fabrica en la Región de los Ríos en el estado de Tabasco, México, que comprende los municipios de Jonuta, Tenosique, Emiliano Zapata y Balancán, siendo este último en donde tiene mayor arraigo e importancia económica ya que el saber-hacer (know how) tradicional de su proceso forma parte del patrimonio cultural que hacen de este alimento un producto genuino y artesanal, con gran aceptación en el mercado local, estatal y regional; la historia de este queso se puede remontar hasta más de 60 años atrás.

La producción de queso de poro se lleva a cabo en pequeñas empresas familiares que basan en esta ocupación su sustento, a la vez que generan fuentes de empleo a lo largo de la cadena agroindustrial de la leche, esto es: ganaderos, productores de leche, comerciantes de insumos y distribuidores del queso. Debido a la importancia económica que tiene el queso de Poro y las empresas que lo elaboran, han sido objeto de múltiples investigaciones en donde se busca solucionar las diversas problemáticas que, por su condición de producto artesanal y la tradición en su elaboración.

Sin embargo, no existen estudios para determinar cómo están estructurados los costos en las empresas que producen y comercializan el queso de poro, de tal manera que los precios de venta actuales se establecen mediante estimaciones a grosso modo, sin tener certeza del margen de utilidad.

Por tal motivo, resulta imperativo realizar estudios que generan datos precisos sobre los costos reales del queso de poro y de los otros productos que ofertan estas empresas, todo esto contribuirá a que los productores tomen mejores decisiones respecto al establecimiento de precios de venta, capacidad de endeudamiento e inversiones. Por otra parte, los resultados obtenidos constituyen el insumo para otros estudios, $v$. gr. el análisis financiero, costos de los activos diferidos intangibles, capacidad de endeudamiento, entre otros.

Actualmente, sólo se tiene un costo aproximado de la producción basado en la cuantificación de los costos directos más evidentes, pero no se toman en cuenta los otros costos que inciden directamente en el margen de utilidad de la empresa: diferenciación de los costos por producto, ingreso por la venta del suero, gastos por concepto de gasolina, luz, agua -recursos empleados de manera indistinta en la empresa y familia-, costos de la depreciación de los activos, entre otros.

Con base a lo anterior, surge las preguntas de investigación: ¿Cuál es el costo real por kilogramo de cada producto que se produce en la empresa? ¿Cuál es la estructura de costos de la empresa? ¿Cuál es el margen de utilidad neta y el de comercialización? ¿Los precios de venta actuales son los adecuados? Por lo tanto, es necesario proporcionar respuestas mediante una investigación planificada que permita obtener datos confiables para conocer la estructura de costos de los diferentes productos que se producen y comercializan en la Quesería El Bejucal.

\section{NotAS DE AUTOR}




\section{Materiales y MÉtodo}

El estudio se desarrolló en la empresa denominada Quesería El Bejucal fundada en el año 1968, con Régimen Intermedio como persona física, además en el año 2012 además es beneficiaria de la certificación Marca Colectiva bajo el nombre "Queso de Poro Genuino de Balancán, Región de Origen", distinción geográfica otorgada por el Instituto Mexicano de Protección Industrial (IMPI, 2015). Es una empresa familiar ya que en ella laboran 4 personas que tienen parentesco directo, en donde la familia tiene el control y la administra, manteniendo la expectativa de que la empresa continúe de una generación a otra.

Se utilizó la metodología de estudio de caso descrita por Martínez (2006) a partir de Shaw (1999), tomando en consideración que esta autora establece que el método de estudio de caso es conveniente para temas prácticamente nuevos, y porque Chetty (1996) afirma que dicho método tiene rigurosidad científica, principio básico para tener certeza en los resultados.

En primer lugar, se hizo el planteamiento del problema, las preguntas de investigación y los objetivos del estudio. Seguidamente se realizó una revisión bibliográfica para conocer el acervo relativo al tema, no encontrando estudios referentes a la estructura de costos en empresas que elaboran queso de poro.

Para la consecución de esta investigación se empleó el método de estudio de caso, clasificada dentro del enfoque cualitativo debido a las características per se de la empresa objeto del estudio, en donde las respuestas a las preguntas de investigación se obtienen a partir de los hechos que ocurren en tiempo real y los datos se recolectan a partir de observaciones, cuestionarios y entrevistas. No se manipulan variables, antes bien, se busca entender la realidad, de ahí el enfoque interpretativo e inductivo, o sea, va de lo particular a lo general.

Otra característica del enfoque cualitativo es que no hace uso de la estadística para obtener una muestra representativa, sino que se puede involucrar a unos pocos sujetos porque no se pretende generalizar los resultados del estudio, aunque si se pueden extrapolar (Hernández et al., 2006).

Estos mismos autores definen al estudio de caso como estudios que al utilizar los procesos de investigación cuantitativa, cualitativa o mixta; analizan profundamente una unidad para responder al planteamiento del problema, probar hipótesis y desarrollar alguna teoría (SNIM, (2017).

Al plantear la investigación como un estudio de caso, los resultados obtenidos pueden ser extrapolados a otras empresas similares. Por tal motivo, se tomó a la empresa Quesería El Bejucal como una entidad representativa ya que: a) es una empresa familiar; b) los niveles de producción son pequeños y similares a otras empresas ubicadas en la región; c) el área de producción y el área de ventas se ubican en el mismo domicilio familiar; d) además del queso de poro producen otros quesos; e) pertenece a la SPR "Queso de Poro Genuino de Balancán”, f) el proceso de elaboración del queso es similar al de las otras empresas y, por último, los dueños mostraron interés y disponibilidad para el desarrollo del estudio para conocer la estructura de sus costos, D.O.F. (2018),Enciclopedia de los Municipios y Delegaciones de México (2017), SIGPI, (2015).

El conocimiento de los principales criterios técnicos y financieros es fundamental para recabar la información, por esto se determinaron los parámetros de producción, precio a considerar de los insumos, maquinaria empleada y principales fuentes de información de precios para que los costos sean consistentes y confiables. Para tal fin, fue necesario establecer un cuestionario que permita el correcto levantamiento de datos. Para conocer la estructura de costos de producción de queso de poro, se utilizó una metodología adaptada a partir de criterios y aspectos teoricos de Gittinger (1987); Muñante (2002); la propuesta de Fideicomisos Instituidos con Relación a la Agricultura: Elaboración de Costos de Producción Agrícola (FIRA, 2009), y del planteamiento de Baca (2006). 


\section{ANÁLISIS DE RESULTADOS}

Según Miller y Le-Breton (2005), citado por Romero (2006), se trata de una empresa familiar ya que en ella laboran 4 personas que tienen parentesco directo, en donde la familia tiene el control y administra manteniendo la expectativa de que la empresa continúe de una generación a otra.

Los productos que elaboran son: queso de poro principalmente y en menor proporción: queso panela, queso crema, queso de hebra (conocido como queso Oaxaca o quesillo) y queso cincho. La empresa tiene una capacidad instalada de $700 \mathrm{~L}$ de leche/día, pero actualmente sólo se están procesando $350 \mathrm{~L}$ por ser temporada baja. En temporada alta, procesan $600 \mathrm{~L}$ de leche/día en sus diferentes productos.

Para la venta, la empresa ha establecido dos precios, dependiendo si es a mayoreo o a menudeo (Cuadro 1), la diferencia es de $\$ 30.00$ en el caso del queso de poro y el queso cincho, y de $\$ 20.00$ en el caso de los otros quesos.

\section{CUADRO 1}

Precios al mayoreo y menudeo de la Quesería Bejucal

\begin{tabular}{lll}
\hline Producto $(\mathrm{Kg})$ & $\begin{array}{l}\text { Precio Mayoreo } \\
(\$)\end{array}$ & $\begin{array}{l}\text { Precio menudeo } \\
(\$)\end{array}$ \\
\hline Queso de poro & 140 & 170 \\
Queso Oaxaca & 80 & 100 \\
Queso panela & 80 & 100 \\
Queso crema & 80 & 100 \\
Queso cincho & 90 & 120 \\
Suero (L) & & 0.25 \\
\hline
\end{tabular}

elaboración propia, basado en la entrevista al productor

Cabe señalar que los precios de venta de los productos, principalmente del queso de poro, varían de acuerdo al lugar en que se expende. Por ejemplo, la pieza de queso de poro de aproximadamente $350 \mathrm{gr}$ se expende en el aeropuerto hasta en $\$ 70.00$ mientras que en los mercados públicos y tiendas se vende a un precio promedio de $\$ 50.00$.

\section{Estructura de costos}

En la Quesería El Bejucal se labora los siete días de la semana, aunque no se elaboran todos los tipos de quesos todos los días, excepto el queso de poro ya que es el que tiene mayor demanda, para el cual se cuaja leche todos los días. Por tal razón, se tomará la semana como base de cálculo. Al ser una empresa familiar y al estar ubicadas -la casa habitación y la planta de producción en el mismo predio, la jornada laboral se adecúa a la cantidad de leche cuajada en el día, a los productos que se elaboran y a los compromisos contraídos para la entrega de los productos, ya que los responsables directos de la producción: el maestro quesero y el encargado de la producción, son padre e hijo, respectivamente.

Como se especificó en el párrafo anterior, se tomará la semana como base de cálculo para obtener los costos anualmente. Los datos se recolectaron en la semana correspondiente del 21 al 27 de Junio de 2015, que corresponde a la temporada baja. En el Cuadro 2, se presenta los litros procesados, cantidad de Kg de cada tipo de queso obtenido (el porcentaje que significa), el rendimiento, y los Kg comercializados al mayoreo y al menudeo. Del total de la leche, se destina el $64.2 \%$ para la producción de queso de poro, seguido del queso de hebra para el cual se destina 18.3\%. Los otros tipos de queso se producen sólo en pequeñas cantidades: $5.5 \%$ para el queso crema, 4.6\% para el queso panela y $7.5 \%$ para el queso cincho. Por tal razón, al hacer los cálculos para determinar la estructura de costos, se tendrá en cuenta estas proporciones. 
Del total de la leche, se destina el $64.2 \%$ para la producción de queso de poro, seguido del queso de hebra para el cual se destina 18.3\%. Los otros tipos de queso se producen sólo en pequeñas cantidades: $5.5 \%$ para el queso crema, $4.6 \%$ para el queso panela y $7.5 \%$ para el queso cincho. Por tal razón, al hacer los cálculos para determinar la estructura de costos, se tendrá en cuenta estas proporciones.

Por otra parte, como se puede apreciar en el Cuadro 2, sólo el queso de poro se comercializa a través de algún distribuidor: $36.5 \%$ que corresponde a $23 \mathrm{Kg}$. La producción de este tipo de queso es la ocupación fundamental ya que corresponde al $59 \%$ de la producción total. El resto de los tipos de queso, se comercializan directamente al consumidor en el área de venta de la empresa, aunque ocasionalmente se comercializan vía distribuidor o se procesan mayor número de Kg, previo pedido; por tal motivo los precios están especificados.

CUADRO 2.

Producción en la Quesería El Bejucal

\begin{tabular}{|c|c|c|c|c|c|c|c|c|}
\hline $\begin{array}{l}\text { Tipo } \\
\text { de } \\
\text { queso }\end{array}$ & $\begin{array}{l}\text { Volumen } \\
\text { de } \\
\text { leche (L) }\end{array}$ & $\%$ & $\begin{array}{l}\text { Total de } \\
\text { queso } \\
\text { obtenido } \\
\text { (Kg) }\end{array}$ & $\begin{array}{l}\text { \% de la } \\
\text { producción }\end{array}$ & $\begin{array}{l}\text { Rendimiento } \\
\text { (\%) }\end{array}$ & $\begin{array}{l}\text { Volumen } \\
\text { de } \\
\text { Suero } \\
\text { (aprox) } \\
\text { (L) }\end{array}$ & $\begin{array}{l}\text { Total de queso } \\
\text { comercializados } \\
\text { de manera } \\
\text { directa }(\mathrm{Kg})\end{array}$ & $\begin{array}{l}\text { Total de } \\
\text { queso } \\
\text { comercializado } \\
\text { mediante } \\
\text { distribuidor } \\
(\mathrm{Kg})\end{array}$ \\
\hline poro & 700 & 64.2 & 63 & 58.8 & 9 & 637 & 23 & 40 \\
\hline hebra & 200 & 18.3 & 22 & 20.5 & 11 & 178 & 22 & 0 \\
\hline crema & 60 & 5.5 & 8 & 7.4 & 13.3 & 52 & 8 & 0 \\
\hline panela & 50 & 4.6 & 6 & 5.6 & 12 & 44 & 6 & 0 \\
\hline cincho & 80 & 7.5 & 8 & 7.4 & 10 & 72 & 8 & 0 \\
\hline Total & 1090 & 100 & 107 & 100 & & 983 & 67 & 40 \\
\hline
\end{tabular}

Elaboración propia a partir de datos obtenidos en la entrevista al productor

Para determinar la estructura de costos fue necesario establecer los precios de los diferentes insumos que se emplean en la manufactura de los productos, mismos que se presentan en el Cuadro 3. Se incluyen los datos de cada proveedor. 
CUADRO 3.

Precios de insumos utilizados en la Quesería Bejucal

\begin{tabular}{|c|c|c|c|c|}
\hline $\begin{array}{l}\text { Materia } \\
\text { prima/Insumo }\end{array}$ & $\begin{array}{l}\text { Unidad } \\
\text { de } \\
\text { medida }\end{array}$ & Proveedor & $\begin{array}{l}\text { Datos del proveedor } \\
\text { (domicilio, Tel, } \\
\text { correo, referencia) }\end{array}$ & $\begin{array}{l}\text { Precio } \\
\$\end{array}$ \\
\hline Leche & $\mathrm{L}$ & $\begin{array}{l}\text { Sr. Belisario } \\
\text { Abreu Sr. } \\
\text { Porfirio Cabrera }\end{array}$ & $\begin{array}{l}\text { Domicilio conocido. } \\
\text { Balancán, Tabasco. }\end{array}$ & 5.00 \\
\hline Cuajo & L & $\begin{array}{l}\text { Veterinaria La } \\
\text { Isla }\end{array}$ & $\begin{array}{l}\text { Av. Gregorio Méndez } \\
\text { SiN. Balancán, Tab. } \\
\text { C.P. } 86930\end{array}$ & 230.00 \\
\hline Sal & $\mathrm{Kg}$ & Donosusa & $\begin{array}{l}\text { Av. Belisario } \\
\text { Dominguez, S /N. Col. } \\
\text { El Carmen, Balancán, } \\
\text { Tab. C.P. } 86930\end{array}$ & 5.75 \\
\hline $\mathrm{CaCl} 2$ & $\mathrm{Kg}$ & Villamex & \multirow{3}{*}{$\begin{array}{l}\text { Calle Cristóbal Colón } \\
\text { S /N. entre Libertad y } \\
\text { Vicente Guerrero, } \\
\text { Col. Centro. E. } \\
\text { Zapata, Tab. }\end{array}$} & 40.00 \\
\hline $\begin{array}{l}\text { Dióxido de } \\
\text { Titanio }\end{array}$ & $\mathrm{Kg}$ & Villamex & & 80.00 \\
\hline Pegamento & L & Villamex & & 40.00 \\
\hline Papel celofán & Pliego & Optivosa & $\begin{array}{l}\text { Av. Adolfo Ruiz } \\
\text { Cortínes No. } 2105 \\
\text { por Av. } 27 \text { de } \\
\text { Febrero. Col. Atasta. } \\
\text { Villahermosa, } \\
\text { Tabasco, México. } \\
\text { C.P. } 86100 \text { Tel. (993) } \\
\text { 310-31-35 }\end{array}$ & 4.12 \\
\hline Etiquetas & Tabloide & $\begin{array}{l}\text { Digital Copy } \\
\text { Click }\end{array}$ & $\begin{array}{l}\text { Zacatenango } 121 \text {, } \\
\text { Progresivo Ciudad } \\
\text { Industrial. } \\
\text { Villahermosa, Tab. } \\
\text { C.P. } \\
86017 \\
\text { Tel. (993) } 3530306\end{array}$ & 8.50 \\
\hline Acido Acético & L & La Puerta del Sol & $\begin{array}{l}\text { Av. Francisco I. } \\
\text { Madero. } \\
\text { Villahermosa, Tab. }\end{array}$ & 50.00 \\
\hline Cera & $\mathrm{Kg}$ & Multicera & $\begin{array}{l}\text { Rectángulos } 100 . \\
\text { Arco Vial. García, } \\
\text { Nuevo León. C.P. } \\
66023 \text { Tel. } \\
8181210100\end{array}$ & 85.00 \\
\hline Bolsas & Rollo & $\begin{array}{l}\text { Comercializadora } \\
\text { Sánchez }\end{array}$ & $\begin{array}{l}\text { Av. Gregorio Méndez } \\
\text { ezq Av. La Paz. } \\
\text { Balancán, Tab. C.P. } \\
86930\end{array}$ & 30.00 \\
\hline
\end{tabular}

Elaboración propia a partir de datos obtenidos en la entrevista al productor

\section{Costos directos de fabricación}

Para calcular el costo de materia prima e insumos de la producción, se considerará cada tipo de queso que produce la empresa (de poro, panela, crema, cincho y de hebra). En la Quesería Bejucal, no se produce todos los tipos de queso todos los días, sino que engloba la producción de manera semanal, a excepción del queso de Poro que se produce a diario. El costo de producción del queso de poro se presenta en el Cuadro 4. 
Revista Iberoamericana de Bioeconomía y Cambio Climático, 2019, 5(10), Agosto-Diciembre, ISSN: 241...

CUADRO 4.

Costo directo de producción del queso de poro

\begin{tabular}{llllll}
\hline Concepto & $\begin{array}{l}\text { Unidad de } \\
\text { medida }\end{array}$ & $\begin{array}{l}\text { Consumo } \\
\text { Semanal }\end{array}$ & Costo $(\$)$ & Consumo anual & $\begin{array}{l}\text { Costo del consumo anual } \\
\text { total }(\$)\end{array}$ \\
\hline Leche & $\mathrm{L}$ & 700 & 5.00 & $36,400.00$ & $182,000.00$ \\
Cuajo & $\mathrm{L}$ & 0.012 & 230.00 & 4.37 & $1,004.64$ \\
Sal & $\mathrm{Kg}$ & 1 & 5.75 & 364.00 & $2,093.00$ \\
Etiquetas & $\mathrm{Tabloide}$ & 2 & 8.50 & 728.00 & $6,188.00$ \\
Cera & $\mathrm{Kg}$ & 1.08 & 85.00 & 393.12 & $33,415.20$ \\
Celofán & Pliego & 3 & 4.12 & $1,092.00$ & $4,499.04$ \\
Pegamento & $\mathrm{L}$ & & 40.00 & 4.00 & 160.00 \\
Total & & & & & $229,359.88$ \\
Costo unitario & & & & & 70.01 \\
\hline
\end{tabular}

Elaboración propia, basado en la entrevista al productor.

Se aprecia un mayor costo directo respecto al costo directo de los otros tipos de quesos: queso crema (Cuadro 5), queso panela (Cuadro 6), queso de hebra (Cuadro 7 ) y queso cincho (Cuadro 8).

CUADRO 5.

Costo directo de producción del queso crema

\begin{tabular}{llllll}
\hline Concepto & $\begin{array}{l}\text { unidad de } \\
\text { medida }\end{array}$ & $\begin{array}{l}\text { Consumo } \\
\text { semanal }\end{array}$ & Costo $(\$)$ & Consumo anual & $\begin{array}{l}\text { Consumo anual total en } \\
\text { pesos. }\end{array}$ \\
\hline Leche & $\mathrm{L}$ & 60 & 5.00 & 3120 & $15,600.00$ \\
Cuajo & $\mathrm{L}$ & 0.012 & 230.00 & 4.368 & $1,004.64$ \\
Sal & $\mathrm{Kg}$ & 0.4 & 5.75 & 145.6 & 837.20 \\
Total & & & & & $17,441.84$ \\
Costo Unitario & & & & & 41.93 \\
\hline
\end{tabular}

laboración propia, basado en la entrevista al productor

CUADRO 6.

Costo directo de producción del queso panela

\begin{tabular}{llllll}
\hline Concepto & $\begin{array}{l}\text { unidad de } \\
\text { medida }\end{array}$ & $\begin{array}{l}\text { Consumo } \\
\text { semanal }\end{array}$ & Costo $(\$)$ & Consumo anual & $\begin{array}{l}\text { Consumo anual total en } \\
\text { pesos }(\$)\end{array}$ \\
\hline Leche & $\mathrm{L}$ & 50 & 5.00 & $2,600.00$ & $13,000.00$ \\
Cuajo & $\mathrm{L}$ & 0.006 & 230.00 & 0.31 & 71.76 \\
Cacl2 & $\mathrm{Kg}$ & 0.01 & 40.00 & 0.52 & 20.80 \\
$\begin{array}{l}\text { Dióxido de } \\
\text { Titano }\end{array}$ & $\mathrm{Kg}$ & 0.01 & 80.00 & 0.52 & 41.60 \\
Sal & $\mathrm{Kg}$ & 0.1 & 5.75 & 5.20 & 29.90 \\
Total & & & & & $13,164.06$ \\
Costo Unitario & & & & & 42.19 \\
\hline
\end{tabular}

Elaboración propia, basado en la entrevista al productor 
CUADRO 7.

Costo directo de producción del queso de hebra

\begin{tabular}{llllll}
\hline Concepto & $\begin{array}{l}\text { unidad de } \\
\text { medida }\end{array}$ & $\begin{array}{l}\text { Consumo } \\
\text { semanal }\end{array}$ & Costo $(\$)$ & Consumo anual & $\begin{array}{l}\text { Consumo anual total en } \\
\text { pesos }(\$)\end{array}$ \\
\hline Leche & $\mathrm{L}$ & 200 & 5.00 & $10,400.00$ & $52,000.00$ \\
Cuajo & $\mathrm{L}$ & 0.024 & 230.00 & 1.25 & 287.04 \\
CaCl2 & $\mathrm{Kg}$ & 0.04 & 40.00 & 2.08 & 83.20 \\
Dióxido de titano & $\mathrm{Kg}$ & 0.04 & 80.00 & 2.08 & 166.40 \\
Sal & $\mathrm{Kg}$ & 1 & 5.75 & 52.00 & 299.00 \\
Total & & & & & $52,835.64$ \\
Costo unitario & & & & & 46.19 \\
\hline
\end{tabular}

Elaboración propia, basado en la entrevista al productor

\section{CUADRO 8.}

Costo directo de producción del queso cincho

\begin{tabular}{lllrll}
\hline Concepto & $\begin{array}{l}\text { unidad de } \\
\text { medida }\end{array}$ & $\begin{array}{l}\text { Consumo } \\
\text { semanal }\end{array}$ & \multicolumn{1}{c}{$\begin{array}{l}\text { Costo } \\
\text { Consumo anual }\end{array}$} & $\begin{array}{l}\text { Consumo anual total en } \\
\text { pesos. }\end{array}$ \\
\hline Leche & $\mathrm{L}$ & 80 & 5.00 & $4,160.00$ & $20,800.00$ \\
Cuajo & $\mathrm{L}$ & 0.006 & 230.00 & 0.31 & 71.76 \\
Chile Guajillo & $\mathrm{Kg}$ & 0.01 & 100.00 & 0.52 & 52.00 \\
Sal & $\mathrm{Kg}$ & 0.35 & 5.75 & 18.20 & 104.65 \\
Total & & & & & $21,028.41$ \\
Costo unitario & & & & & 50.55 \\
\hline
\end{tabular}

Elaboración propia, basado en la entrevista al productor.

Dentro de los costos directos de fabricación se encuentra el costo derivado de la mano de obra, o sea del personal que trabaja directamente en la manufactura (elaboración o producción) del bien, en este caso, de los diferentes tipos de quesos, dicho costo se muestra en el Cuadro 9.

CUADRO 9.

Costo de mano de obra directa

\begin{tabular}{lllll}
\hline Puesto & $\begin{array}{l}\text { Número } \\
\text { de } \\
\text { plazas }\end{array}$ & $\begin{array}{l}\text { Condición } \\
\text { laboral }\end{array}$ & $\begin{array}{l}\text { Sueldo } \\
\text { mensual } \\
(\$)\end{array}$ & $\begin{array}{l}\text { Sueldo } \\
\text { anual (\$) }\end{array}$ \\
\hline Auxiliar & 1 & $\begin{array}{l}\text { lunes - } \\
\text { Sábado }\end{array}$ & $2,800.00$ & $33,600.00$ \\
Mtro Quesero & 1 & $\begin{array}{l}\text { Lunes - } \\
\text { Domingo }\end{array}$ & $5,200.00$ & $62,400.00$ \\
$\begin{array}{l}\text { Encargado de } \\
\text { Producción }\end{array}$ & 1 & $\begin{array}{l}\text { Lunes - } \\
\text { Domingo }\end{array}$ & $5,200.00$ & $62,400.00$ \\
Total & & & $3,200.00$ & $158,400.00$ \\
\hline
\end{tabular}

Elaboración propia, basado en la entrevista al productor.

$\mathrm{Al}$ ser una quesería, se trabaja todos los días de la semana, por lo tanto, el encargado de la producción y el maestro quesero, laboran todos los días; el auxiliar descansa los domingos. Con los costos de la materia prima e insumos (Cuadros 4-8), y los costos de la mano de obra que interviene en la manufactura del producto (Cuadro 9), se calcula el total de los costos directos de producción, que se presenta en el Cuadro 10. 
CUADRO 10.

Costo directo de producción

\begin{tabular}{ll}
\hline Costo Directos & \\
Materia Prima e Insumos & $\begin{array}{l}\text { Importe } \\
\text { anual }(\$)\end{array}$ \\
por producto & $229,359.88$ \\
Queso de Poro & $17,441.84$ \\
Queso Crema & $13,164.06$ \\
Queso Panela & $52,835.64$ \\
Queso de hebra & $21,028.41$ \\
Queso Cincho & $158,400.00$ \\
Mano de Obra & $492,229.83$ \\
\hline Total & \\
\hline
\end{tabular}

Elaboración propia, basado en la entrevista al productor.

\section{Costos indirectos de fabricación}

Para determinar los costos indirectos de fabricación se tomaron en cuenta algunas consideraciones, producto de la entrevista al Ing. Emilio Castro Ehuan, encargado de producción de la empresa: a) respecto a las prestaciones y dividendos a los trabajadores, se otorga una semana de sueldo por concepto de reparto de utilidades y una semana como el equivalente al aguinaldo, este beneficio es sólo para el auxiliar de producción: $\$ 1,400$ al año; b) respecto a los impuestos, la empresa paga $\$ 652.00$ anualmente.

En lo referente a la depreciación de la maquinaria, equipo, vehículo y otros utensilios, se encontró que la mayoría de éstos se encuentran ya depreciados (necesidad de reinversión), pero todavía son utilizados en el acopio de leche, en la producción de quesos y en la comercialización. Los activos que son objeto de depreciación se muestran en el Cuadro 11. 
CUADRO 11.

Inversión en activo fijo y depreciación

\begin{tabular}{|c|c|c|c|c|c|}
\hline Concepto & Cantidad & $\begin{array}{l}\text { Precio } \\
\text { unitario } \\
(\$)\end{array}$ & Costo $(\$)$ & $\begin{array}{l}\text { Vida } \\
\text { útil } \\
\text { en } \\
\text { años }\end{array}$ & $\begin{array}{l}\text { Depreciación } \\
\text { anual }(\$)\end{array}$ \\
\hline Terreno & & & $150,000.00$ & & \\
\hline Construcción & & & $200,000.00$ & 20 & $10,000.00$ \\
\hline $\begin{array}{l}\text { Camioneta } \\
\text { Pick up para } \\
\text { colecta de } \\
\text { leche }\end{array}$ & 1 & $60,000.00$ & $60,000.00$ & & \\
\hline $\begin{array}{l}\text { Mesas de } \\
\text { trabajo }\end{array}$ & 2 & $18,000.00$ & $36,000.00$ & & \\
\hline Lecheras & 2 & 500.00 & $1,000.00$ & & \\
\hline $\begin{array}{l}\text { Tinas de } \\
\text { Cuajada }\end{array}$ & 4 & $15,000.00$ & $60,000.00$ & & \\
\hline Balanzas & 2 & $1,000.00$ & $2,000.00$ & & \\
\hline Cuchillos & 5 & 20.00 & 100.00 & & \\
\hline Moldes & 200 & 250.00 & $50,000.00$ & 10 & $5,000.00$ \\
\hline Contrapesos & 60 & 3.00 & 180.00 & & \\
\hline Palancas & 60 & 4.00 & 240.00 & & \\
\hline $\begin{array}{l}\text { moldes para } \\
\text { queso cincho }\end{array}$ & 15 & 40.00 & 600.00 & 10 & 60.00 \\
\hline $\begin{array}{l}\text { Moldes para } \\
\text { queso panela }\end{array}$ & 50 & 35.00 & $1,750.00$ & 3 & 583.33 \\
\hline Sillas & 1 & 400.00 & 400.00 & & \\
\hline $\begin{array}{l}\text { Prensa } \\
\text { Holandesa }\end{array}$ & 1 & $15,000.00$ & $15,000.00$ & & \\
\hline $\begin{array}{l}\text { Balanza } \\
\text { manual }\end{array}$ & 1 & $1,200.00$ & $1,200.00$ & & \\
\hline $\begin{array}{l}\text { Tanque de } \\
\text { gas }\end{array}$ & 1 & 700.00 & 700.00 & 10 & 70.00 \\
\hline \multicolumn{6}{|l|}{$\begin{array}{l}\text { Activo fijo en } \\
\text { el área de } \\
\text { ventas }\end{array}$} \\
\hline $\begin{array}{l}\text { Refrigerador } \\
\text { vertical }\end{array}$ & 1 & $18,000.00$ & $18,000.00$ & 10 & $1,800.00$ \\
\hline $\begin{array}{l}\text { Refrigerador } \\
\text { horizontal }\end{array}$ & 1 & $23,000.00$ & $23,000.00$ & 10 & $2,300.00$ \\
\hline $\begin{array}{l}\text { Automovil } \\
\text { compacto }\end{array}$ & 1 & & $140,000.00$ & 5 & $28,000.00$ \\
\hline Total & & & & & $47,813.33$ \\
\hline
\end{tabular}

Elaboración propia, basado en la entrevista al productor.

Se considera la depreciación del automovil en un plazo de 5 años, ya que es el tiempo en el cual la empresa pagará ese bien, aunque de manera contable la depreciación debería ser a 3 años. Se considera el precio factura, ya que los intereses del mismo se verán reflejados en el flujo de efectivo como costos financieros que se restan a la utilidad bruta.

Dentro del costeo es importante considerar los costos que representan los materiales de limpieza, desinfección e higiene, ya que son fundamentales para observar la Buenas Prácticas de Fabricación, tal como lo estipula la NOM-SSA1-251-2009 (D.O.F., 2018). Dichos costos se muestran en el Cuadro 12. 
CUADRO 12.

Costos de materiales de limpieza, desinfección e higiene

\begin{tabular}{llllcc}
\hline Concepto & $\begin{array}{l}\text { Unidad de } \\
\text { medida }\end{array}$ & $\begin{array}{l}\text { Consumo } \\
\text { mensual }\end{array}$ & Consumo anual & Costo unitario (\$) & Costo anual (\$) \\
\hline $\begin{array}{l}\text { Cubre boca } \\
\text { desechables }\end{array}$ & $\begin{array}{l}\text { paquete }(10 \\
\text { pzas) }\end{array}$ & 4 & 48 & 10.00 & 480.00 \\
Cofias & Pza & & 4 & 40.00 & 160.00 \\
Mandiles & Pza & & 8 & 65.00 & 520.00 \\
botas de hule & Par & & 4 & 130.00 & 520.00 \\
Manta & $\mathrm{m}$ & & 10 & 55.00 & 550.00 \\
Detergente & $\mathrm{Kg}$ & 4 & 48 & 22.00 & $1,056.00$ \\
Desinfectante & $\mathrm{L}$ & 2 & 24 & 12.00 & 288.00 \\
Escobas & $\mathrm{Pza}$ & & 2 & 30.00 & 60.00 \\
Escurridores & $\mathrm{Pza}$ & & 2 & 40.00 & 80.00 \\
Cepillos & $\mathrm{Pza}$ & & 6 & 10.00 & 60.00 \\
Bolsas & Rollo & 4 & 48 & 30.00 & $1,440.00$ \\
Total & & & & & $5,214.00$ \\
\hline
\end{tabular}

Elaboración propia, basado en la entrevista al productor.

Respecto a los costos derivados del mantenimiento necesario para la buena marcha de la empresa, se consideran 4 eventos al año los cuales pueden ser: reparación de equipo de refrigeración, reparación de vehículos, limpieza del drenaje, ya que son las más representativas. Estos costos se muestran en el Cuadro 13.

CUADRO 13.

Costos por mantenimiento

\begin{tabular}{llll}
\hline Concepto & Evento por año & $\begin{array}{l}\text { costo por evento } \\
(\$)\end{array}$ & Costo anual $(\$)$ \\
$\begin{array}{l}\text { Reparación de equipo de } \\
\text { refrigeración }\end{array}$ & 4 & 500.00 & $2,000.00$ \\
Reparación de vehículo & 4 & 500.00 & $2,000.00$ \\
Mantenimiento de vehículo & 1 & $1,000.00$ & $1,000.00$
\end{tabular}

Elaboración propia, basado en la entrevista al productor.

Otro costo indirecto de fabricación de esta empresa, es el costo derivado del control de calidad de los productos. Dicho costo sólo está integrado por los insumos necesarios para que el personal realice los análisis correspondientes y se detallan en el Cuadro 14.

\section{CUADRO 14.}

Costo por concepto de control de calidad

\begin{tabular}{lllrr}
\hline Insumo & $\begin{array}{l}\text { Unidad de } \\
\text { medida }\end{array}$ & $\begin{array}{l}\text { Consumo } \\
\text { anual }\end{array}$ & Precio Unitario $(\$)$ & $\begin{array}{l}\text { Costo anual } \\
(\$)\end{array}$ \\
\hline $\mathrm{NaOH}$ & $\mathrm{L}$ & 2 & 60.00 & 120.00 \\
\hline Fenolftaleina & $\mathrm{L}$ & 0.25 & 200.00 & 50.00 \\
Alcohol & $\mathrm{L}$ & 2 & 25.00 & 50.00
\end{tabular}

Elaboración propia, basado en la entrevista al productor.

Para calcular el costo del combustible se toma en cuenta la gasolina que ocupa el vehículo en ir a recoger la leche hasta los ranchos y para la compra de insumos; mientras que se considera el gas que se emplea en hervir los moldes y otros utensilios que se usan en el área de producción, estos costos se presentan en el Cuadro 15. 
CUADRO 15.

Costo de combustible

\begin{tabular}{lll}
\hline Combustible & $\begin{array}{l}\text { Costo } \\
\text { mensual }(\$)\end{array}$ & costo anual (\$) \\
\hline Gas butano & 200.00 & $2,400.00$ \\
Gasolina & $2,600.00$ & $31,200.00$ \\
Total & $2,800.00$ & $33,600.00$ \\
\hline
\end{tabular}

Elaboración propia, basado en la entrevista al productor.

En lo que respecta al costo de la energía eléctrica y del suministro de agua potable, se tiene que el costo de la energía eléctrica bimestral es de $\$ 300.00$ lo que equivale a $\$ 1,800.00$ anuales y el costo del servicio del agua potable proveniente de la red municipal es de $\$ 30.00 \mathrm{al}$ mes, lo que representa un total de $\$ 360.00 \mathrm{al}$ año. Con todos los datos anteriores se calcula el total de los costos indirectos, mismos que se presenta en el siguiente Cuadro 16.

\section{CUADRO 16}

Total de Costos Indirectos

\begin{tabular}{ll}
\hline Costos indirectos (\$) & \\
\hline $\begin{array}{l}\text { Dividendo a los } \\
\text { trabajadores }\end{array}$ & $1,400.00$ \\
Impuestos & $7,824.00$ \\
Depreciación & $47,813.33$ \\
Material de Limpieza y & $5,214.00$ \\
Desinfección & \\
Mantenimiento & $5,500.00$ \\
Control de calidad & 220.00 \\
Combustibles & $33,600.00$ \\
Energía eléctrica & $1,800.00$ \\
Agua & 360.00 \\
Total & $94,507.33$ \\
\hline
\end{tabular}

Elaboración propia, basado en la entrevista al productor.

\section{Costo de administración y ventas.}

Para calcular los costos de administración se consideran los materiales y servicios empleados en las oficinas de administración, éstos se muestran en el Cuadro 17

\section{CUADRO 17.}

Costo de administración

\begin{tabular}{llcll}
\hline Concepto & Unidad & Costo $(\$)$ & Consumo anual & $\begin{array}{l}\text { Costo } \\
\text { anual (\$) }\end{array}$ \\
\hline Lapiceros & Pza & 5.00 & 10 & 50.00 \\
Libretas & Pza & 8.00 & 2 & 16.00 \\
Teléfono & renta mensual & 200.00 & 12 & $2,400.00$ \\
Contabilidad & servicio & 450.00 & 12 & $5,400.00$ \\
& mensual & & &
\end{tabular}

Elaboración propia, basado en la entrevista al productor. 
Respecto al gasto de ventas, se toma en cuenta al personal que está directamente al frente del mostrador y se encarga de la atención a clientes y de despachar los pedidos. Otros gastos que se toman en cuenta son los gastos generados por combustibles, peajes y viáticos al hacer la labor de ventas. Para el caso específico de esta empresa, estos gastos se generan al trasladar los productos a la ciudad de Villahermosa, Tabasco, principalmente. Estos gastos se presentan en el Cuadro 18.

CUADRO 18.

Gasto de ventas

\begin{tabular}{lccl}
\hline Puesto & Número de plazas & $\begin{array}{l}\text { Sueldo mensual } \\
(\$)\end{array}$ & Sueldo Anual (\$) \\
$\begin{array}{lcc}\text { Responsable de } \\
\text { ventas }\end{array}$ & 1 (Lun - Dom) & $3,200.00$ & $38,400.00$ \\
Otros gastos & Gasto semanal & Gasto anual \\
Gasolina & 300.00 & $15,600.00$ \\
Peaje & 40.00 & $2,080.00$ \\
& - & \\
& Elaboración propia, basado en la entrevista al productor
\end{tabular}

Con los gastos de administración y los gastos generados en la distribución y venta, se tienen los gastos de administración y ventas (en algunos casos se conoce como gastos del periodo), que se presentan en el Cuadro 19.

\section{CUADRO 19.}

Gastos de administración y ventas

\begin{tabular}{lc}
\hline \multicolumn{2}{l}{ Gastos de administración y ventas $(\$)$} \\
\hline Materiales de & $7,866.00$ \\
administración & \\
Gastos de ventas & $58,680.00$ \\
Total & $66,546.00$ \\
\hline
\end{tabular}

Elaboración propia, basado en la entrevista al productor.

Con todos los costos anteriores, se calcula el costo total de producción de la empresa, este es la estructura de costos, tal como se muestra en el Cuadro 20.

CUADRO 20.

Costo total de producción en pesos (estructura de costos)

\begin{tabular}{ll}
\hline Costos directos & $492,229.83$ \\
\hline Costos Indirectos & $94,507.33$ \\
Gastos del Periodo & $66,546.00$ \\
Total & $653,283.16$
\end{tabular}

Elaboración propia, basado en la entrevista al productor.

\section{Costo unitario de fabricación}

Para determinar el costo unitario de fabricación se toma en cuenta que el costo correspondiente a la materia prima e insumos (costo directo) se divide entre los kg de cada tipo de queso, mientras que los costos indirectos 
y los costos de administración y ventas se dividen entre el total de los $\mathrm{Kg}$ de queso producidos y vendidos. El costo unitario de cada tipo de queso se presenta en el Cuadro 21.

CUADRO 21.

Costo unitario de fabricación

\begin{tabular}{|c|c|c|c|c|}
\hline \multicolumn{5}{|c|}{ Costo unitario de fabricación. } \\
\hline Producto & Costo directo & $\begin{array}{l}\text { Costo indirecto } \\
\text { (\$) }\end{array}$ & $\begin{array}{l}\text { Gastos del } \\
\text { Periodo ( } \$)\end{array}$ & $\begin{array}{l}\text { Costo Unitario } \\
\text { (\$) }\end{array}$ \\
\hline Queso de poro & 70.01 & 16.99 & 11.96 & 98.96 \\
\hline Queso crema & 41.93 & 16.99 & 11.96 & 70.87 \\
\hline Queso panela & 42.19 & 16.99 & 11.96 & 71.14 \\
\hline Queso de hebra & 46.19 & 16.99 & 11.96 & 75.13 \\
\hline 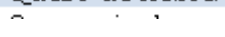 & $\therefore--$ & $\therefore \rightarrow$ & $2 \pi$ & $\rightarrow \cdots$ \\
\hline
\end{tabular}

Se aprecia que el queso de poro tiene el mayor costo unitario de fabricación respecto a los otros tipos de queso, más de $\$ 28$ respecto al queso crema que es el que tiene menos costo y más de $\$ 20$ respecto al queso cincho. Esto se debe a que se usa material de empaque (cera, papel celofán y resistol) y etiqueta, los cuales elevan el costo directo.

\section{Ingresos}

Para calcular los ingresos de la empresa, se toma en cuenta que el $80 \%$ de las ventas se hace a precio de mayoreo, mientras que el $20 \%$ restante se hace directamente al consumidor.

Por otra parte, se toman en cuenta los ingresos por la venta del suero y del recorte del queso de poro. De la entrevista al productor, se sabe que se vende de recorte $\$ 30.00$ en promedio por día, mientras que se venden aproximadamente $200 \mathrm{~L}$ de suero a la semana, lo que equivale \$ 50.00 cada semana. Lo anterior se muestra en el Cuadro 22.

\section{CUADRO 22.}

Presupuesto de Ingresos

\begin{tabular}{|c|c|c|c|c|c|c|}
\hline Producto (Queso) & Producción anual (Kg) & $\begin{array}{l}\text { Precio de venta mayoreo } \\
(\$)\end{array}$ & $\begin{array}{l}\text { Ingresos de ventas mayoreo } \\
(80 \%)\end{array}$ & $\begin{array}{l}\text { Precio de venta } \\
\text { menudeo (\$) }\end{array}$ & $\begin{array}{l}\text { Ingresos de ventas menudeo } \\
\text { (20\%) }\end{array}$ & $\begin{array}{l}\text { Ingresos totales por producto } \\
(\$)\end{array}$ \\
\hline $\begin{array}{l}\text { poro } \\
\text { crema } \\
\text { panela } \\
\text { de hebra } \\
\text { cincho } \\
\text { recorte } \\
\text { suero } \\
\text { Total }\end{array}$ & $\begin{array}{l}3,276 \\
416 \\
312 \\
1,144 \\
416\end{array}$ & $\begin{array}{l}140.00 \\
80.00 \\
80.00 \\
80.00 \\
90.00\end{array}$ & $\begin{array}{l}366,912.00 \\
26,624.00 \\
19,968.00 \\
73,216.00 \\
29,952.00\end{array}$ & $\begin{array}{l}170.00 \\
100.00 \\
100.00 \\
100.00 \\
120.00\end{array}$ & $\begin{array}{l}111,384.00 \\
8,320.00 \\
6,240.00 \\
22,880.00 \\
9,984.00\end{array}$ & $\begin{array}{l}478,296.00 \\
34,944.00 \\
26,208.00 \\
96,096.00 \\
39,936.00 \\
10,200.00 \\
2,600.00 \\
689,000.00\end{array}$ \\
\hline
\end{tabular}

Elaboración propia, basado en la entrevista al productor.

Con los costos totales de producción y los ingresos derivados de las ventas, se calcula la utilidad bruta de la empresa (Cuadro 23). 
CUADRO 23.

Utilidad bruta de la empresa $(\$)$

\begin{tabular}{ll}
\hline Ingresos & $689,000.00$ \\
\hline $\begin{array}{l}\text { Costo total de } \\
\text { fabricación }\end{array}$ & $653,283.16$ \\
Utilidad bruta & $35,716.84$ \\
\hline
\end{tabular}

Elaboración propia, basado en la entrevista al productor.

La Quesería Bejucal debería incrementar su producción para minimizar los costos indirectos y los gastos del periodo en un mayor número de $\mathrm{Kg}$ de queso producidos, ya que en este estudio dichos costos se dividen en pocos $\mathrm{Kg}$ de producto, lo que incide en un alto costo de producción.

Los costos directos están en función de los costos de las materias primas e insumos, por lo que esta empresa debería buscar proveedores con precios más económicos, o establecer acuerdos con otras empresas similares para realizar compras al mayoreo.

El suero es un subproducto que se está desperdiciando, ya que sólo se venden aproximadamente $200 \mathrm{~L}$ a la semana, lo que equivale a $\$ 200$; o sea que quedan aproximadamente $800 \mathrm{~L}$, mismos que se podrían procesar para obtener el requesón como subproducto.

Otro subproducto que no se aprovecha es el líquido resultante del malaxado del queso de hebra, ese líquido es rico en proteínas séricas y grasa que arrastra el agua, por lo que se podría descremar para elaborar crema ácida, mantequilla, base para helado u otros productos.

\section{Discusión}

Villegas y Cervantes (2011), establecen que en México existen más de 40 variedades de quesos genuinos. según Cesín et al. (2012), la problemática de los quesos mexicanos genuinos se puede resumir de la siguiente manera: i) presentan calidad variable desde el punto de vista de composición, sanidad y atributos sensoriales; ii) altos costos de producción, debido a la falta de tecnología apropiada y a la ineficiencia de los procesos de elaboración; iii) problemas de comercialización por los bajos volúmenes de producción y ausencia de estrategias mercadológicas; iv) competencia de los quesos de imitación y quesos extendidos (productos elaborados a partir de sucedáneos de leche, colorantes y saborizantes artificiales); v) falta de cumplimiento con la normatividad, específicamente con la legislación sanitaria, y vi) futuro incierto. En este sentido, se han desarrollado diversos trabajos en el ámbito de la agroindustria láctea artesanal, tales como caracterizaciones (Castañeda et al., 2009), análisis de la cadena de valor (Trejo et al., 2011), de desempeño económico (Melgarejo et al., 2007), y en el ámbito mercadológico (Aristizábal et al., 2000). Por otra parte, al ser una empresa clasificada como PYME (Micro, Pequeña y Mediana Empresa), los estudios que se han realizado para las empresas PYME pueden ser extrapoladas a la quesería objeto de este trabajo, a través de un modelo de transferencia. El modelo desarrollado por Escobar et al. (2009) es interesante ya que valoran una PYME mediante el cálculo de indicadores para determinar la rentabilidad en términos de utilidades. Pero para llevar a cabo esto, se necesitan conocer la estructura de costos para obtener los estados financieros. De igual manera se ha estudiado la competitividad y productividad en empresas familiares o pequeñas y medianas empresas (Romero, 2006), en donde se establece que las relaciones familiares inciden directamente en la empresa, ya sea positiva o negativamente. Respecto a los quesos producidos de manera artesanal en otras partes del mundo, se puede mencionar el caso específico de un estudio que se realizó en las empresas que producen queso Gruyere, en donde se encontró que la manera más sencilla de lograr competitividad con este queso 
es mantener pequeñas fábricas productoras con altos estándares en sus especificaciones de calidad, ya que desde el punto de vista económico, tiene más relevancia la mano de obra que el capital, por lo tanto, no se pueden beneficiar de una economía de escala (Barjolle y Chappuis, 2000, citado por Domínguez et al., 2011). Estudios similares se han desarrollado en empresas de España que producen queso Manchego (Trejo et al., 2011)

En lo que respecta a la apertura de mercados, la competitividad pasa a tener una concepción holística en donde además de la calidad, precio, cantidad producida y las características del producto, se debe tener en cuenta la forma de producir, de distribuir, de llegar al cliente, así como también ser eficiente en el manejo de los recursos monetarios, entendido como manejo financiero (Escobar et al., 2009).

Estos mismos autores establecen que de la información contable se puede extraer la información necesaria para optimizar los recursos; a manera de ejemplo, de los activos se debe extraer aquellos que contribuyen a la generación de rentabilidad, producto de ejercer la actividad principal.

Es por ello que es común que se mida la rentabilidad a partir de las utilidades que se generan al interior de la organización; en otros casos, se podría desarrollar un análisis financiero mediante el cálculo de diferentes índices para conocer la capacidad de operación, liquidez, apalancamiento y otras razones financieras para determinar la rentabilidad de una empresa (Villegas, 2002).

Finalmente tampoco se encontró información de la estructura de costos de queso de poro en las publicaciones del sistema de Agrocostos, una herramienta de FIRA que permite estimar de manera paramétrica costos de producción agrícola en una zona o región determinada bajo una tecnología de producción específica (FIRA, 2018).

\section{Conclusiones}

Se concluye que en los costos directos de producción (sin considerar la mano de obra) de los diferentes tipos de queso, el insumo leche representa un componente significativo, ya que es el de mayor porcentaje en la naturaleza de estos costos. En la ingeniería de costos poco se puede hacer para minimizar este costo, es en el proceso en donde se puede optimizar este recurso mediante el incremento en el rendimiento de producto terminado.

Fue recurrente la importancia del insumo leche para elaborar los diferentes tipos de queso, tan sólo para el queso de poro, el costo directo representó el 79.4\%. El 89.4\% para queso crema. Para el caso del queso de panela éste costo representó el $98.8 \%$ de los costos directos, el patrón se repite en el queso de hebra con el 98.4\% finalmente el queso cincho la leche representa el 98.9\% de los costos directos.

Cuando se considera la mano de obra en el total de los costos directos de producción, ésta representa el $32.2 \%$.

En la partida de costos indirectos se tuvo que el concepto de mayor relevancia fue la depreciación de los activos y el uso de combustibles. En los costos de administración el de mayor importancia es el servicio de contabilidad. En los gastos de venta el rubro más importante es el sueldo del agente de ventas y el combustible (SNIM, (2017).

Según el diagnóstico de costos directos e indirectos y los gastos de administración y ventas se concluye que la asignación de recursos económicos se realiza de forma racional, por lo que presentan normalidad y no se identifica un uso oneroso de los factores de la empresa, por lo que la firma tiene finanzas sanas.

El costo total de producción es equivalente a $\$ 653,283.16$ y el presupuesto de ingresos de la empresa asciende a $\$ 689,000.00$, se infiere una utilidad anual de $\$ 35,716.84$, la utilidad mensual equivale a $\$ 2,976.40$. Entonces por cada peso invertido se ganan 5 centavos, la firma está operando ligeramente por encima del punto de equilibrio. La empresa deberá incrementar el uso de la capacidad instalada para tener mayores niveles de rentabilidad e incluso generar economías de escala. Es pertinente valorar y reflexionar sobre ingresar otro producto lácteo al nicho de mercado que pueda darle mayor robustez financiera a esta quesería. 


\section{REFERENCIAS}

Aristizabal, M.L., Ortiz, L.F., Polanía, H.F., y Ramos, H. (2000). Caso de Estudio: Quesos Andino S.A. Estudios Gerenciales. 75: 67-82.

Baca U., G. 2006. Evaluación de Proyectos. 5ª. ed. McGRAHILL/INTERAMERICANA EDITORES S.A. DE C.V. México. pp. 190 - 217

Castañeda M., T., Boucher, F., Sánchez V., E. y Espinoza O., A. 2009. La concentración de agroindustrias rurales de quesos en el noroeste del Estado de México: un estudio de caracterización. Estudios Sociales. 17(34): 74 - 109.

Cesín-Vargas, A., Cervantes, F., y Villegas de G., A. 2012. La leche y los quesos artesanales en México. Agricultura, Sociedad y Desarrollo. 11(2): 243 - 248. https://doi.org/10.22231/asyd.v11i2.76

Chetty, S. (1996). The case study method for research in small -and médium- sized firms. International small business journal, 15: 73 - 85. https://doi.org/10.1177/0266242696151005

Diario Oficial de la Federación D.O.F. (2018). Norma oficial mexicana NOM-251-SSA1-2009, prácticas de higiene para el proceso de alimentos, bebidas o suplementos alimenticios. Recuperado 18 de junio de 2018, disponible en: http://dof.gob.mx/nota_detalle.php?codigo $=5133449 \&$ fecha $=01 / 03 / 2010$

Domínguez L., A., Villanueva C., A., Arriaga J., C.M., y Espinoza O., A. 2011. Alimentos artesanales y tradicionales: el queso Oaxaca como un estudio del Centro de México. Estudios Sociales. 19(38): 166 - 193.

Enciclopedia de los Municipios y Delegaciones de México (2017). Estado de Tabasco. En http://www.inafed.gob.mx/ work/enciclopedia/EMM27tabasco/municipios/27001a.html. Accesado el 10 de Abril de 2017.

Escobar A., G.E., Arias M., L., y Margarita P., L. 2009. Modelo para medir la situación financiera en empresas PYME. Scientia Et Technica. 15(43): 273 - 278.

Fideicomisos Instituidos en Relación con la Agricultura (FIRA) (2018) Sistema de agrocostos disponible en: https:// www.fira.gob.mx/Nd/Agrocostos.jsp consultado el 09 de junio de 2018.

FIRA (2009) Elaboración de costos de producción agrícolas. 2009. Boletín Informativo. Nueva Época. 4. Fideicomisos Instituidos en Relación con la Agricultura. México.

Gittinger, J.P. (1987). Análisis económico de proyectos agrícolas (2da Ed.). España: Tecno S.A.

Hernández S., R., Fernández C., C., y Baptista L., P. 2006. Metodología de la Investigación. McGRAW-HILL. México. p. 523

Martínez C., P.C., (2006) El método de estudio de caso. Estrategia Metodológica de la Investigación Científica. Pensamiento y Gestión. 20: 166 - 193.

Melgarejo, Z., Arceluz, F. y Simón K. 2007. Desempeño económico: diferencias de pequeñas empresas clasificadas según la estructura de la propiedad del capital. REVESCO. Revista de Estudios Cooperativos. 93: 7 - 38.

Muñante, D. (2002). Seminario en formulación y evaluación de proyectos de inversión (módulo IV y V). Texcoco, Estado de México: División de Ciencias Económico-Administrativas (DICEA) de la Universidad Autónoma Chapingo.

Romero, L.E. (2006). Competitividad y productividad en empresas familiares pymes. Revista Escuela de Administración de Negocios, 57: 131- 141. https://doi.org/10.21158/01208160.n57.2006.378

Sistema de Información de la Gaceta de la Propiedad Industrial, SIGPI, (2015). Instituto Mexicano de la Propiedad Industrial. En http://siga.impi.gob.mx. Accesado el 10 de Abril de 2015.

Sistema Nacional de Información Municipal SNIM, (2017). Disponible en http://www.inafed.gob.mx. Accesado el 10 de Abril de 2017.

Trejo T., B. I., De los Ríos C., I., Figueroa S., B., Gallego M., F.J., y Morales F., F.J. 2011. Análisis de la cadena de valor del queso Manchego en Cuenca, España. Revista Mexicana de Ciencias Agrícolas. 2(4): 545 - 557. https:// doi.org/10.29312/remexca.v2i4.1639

Villegas de G., A. y Cervantes E., F. 2011. La genuinidad y tipicidad en la revalorización de los quesos artesanales mexicanos. Estudios Sociales. 19(38): 146 - 164.

Villegas V., E. 2002. Análisis financiero en los Agronegocios. Revista Mexicana de Agronegocios. 6(10): 337 - 349. 
Ezequiel Paredes-Maas, et al. Análisis de Costos en la Producción de Quesos Artesanales. Estudio d...

Miller, D., \& Le Breton-Miller, I. (2005). Managing for the long run: Lessons in competitive advantage from great family businesses. Harvard Business Press. 